\title{
Perfil Morfológico de Culturistas Brasileiros de Elite em Período Competitivo
}

\author{
Morphological Profile of Elite Brazilian Bodybuilders in \\ Competitive Season
}

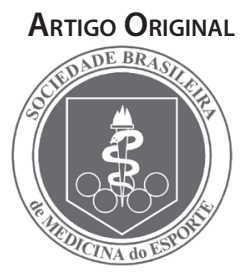

Edilson Serpeloni Cyrino ${ }^{1}$

José Maria Santarém Sobrinho²

Nailza Maestá ${ }^{3}$

Nélson Nardo Jr. ${ }^{4}$

David Augusto dos Reis ${ }^{3}$

Mônica Yara Gabriel Morelli ${ }^{3}$

Roberto Carlos Burini ${ }^{3}$

1. Grupo de Estudo e Pesquisa em Metabolismo, Nutrição e Exercício. Centro de Educação Física e Esporte. Universidade Estadual de Londrina.

2. Centro de Estudos em Ciências da Atividade Física - FM.USP.

3. Centro de Metabolismo, Exercício e Nutrição. Faculdade de Medicina. Universidade Estadual Paulista

- Botucatu/SP.

4. Departamento de Educação Física. Universidade Estadual de Maringá.

Endereço para correspondência: Edilson Serpeloni Cyrino Grupo de Estudo e Pesquisa em Metabolismo, Nutrição e Exercício Centro de Educação Física e Esporte Universidade Estadual de Londrina Rod. Celso Garcia Cid, km 380

- Campus Universitário

CEP 86051-990 - Londrina, PR

- Brasil

E-mail: emcyrino@uel.br

Submetido em 22/09/2006

Versão final recebida em 14/04/2007 Aceito em 17/05/2007

\begin{abstract}
RESUMO
O objetivo deste estudo foi analisar o perfil morfológico de culturistas brasileiros de elite que competiram no XXVII Campeonato Brasileiro de Culturismo-Musculação. Um total de 28 atletas (27,0 \pm 7,4 anos; 78,2 \pm $8,7 \mathrm{~kg} ; 170,1 \pm 6,9 \mathrm{~cm}$ ), do sexo masculino, foram voluntariamente estudados. Medidas antropométricas (massa corporal, estatura, espessura de dobras cutâneas, perímetros e diâmetros) foram coletadas de todos os sujeitos no período da manhã, no dia da competição. Os valores estimados da composição corporal dos atletas foram os seguintes: gordura corporal relativa, 5,8 0,5\%; massa corporal magra, 73,6 \pm 8,2kg; massa muscular, $54,3 \pm 6,7 \mathrm{~kg}$; área muscular do braço, 108,51 \pm 15,19 $\mathrm{cm}^{2}$. Por outro lado, o somatótipo médio encontrado foi: endomorfia, 1,11 \pm 0,29; mesomorfia, 8,26 \pm 0,67; ectomorfia, 0,95 \pm 0,53 (mesomorfo balanceado). Além de os culturistas apresentarem elevados níveis de desenvolvimento muscular ( 70\%) e baixos depósitos de gordura corporal, verificou-se uniformidade nos acúmulos de gordura localizada. A predominância do componente mesomorfo confirmou a estrutura muscular acentuadamente desenvolvida encontrada nos atletas investigados. Portanto, os resultados do presente estudo demonstram que atletas de culturismo de elite apresentam no dia da competição grande volume muscular, bem como excelente definição muscular, proporcionalidade e simetria.
\end{abstract}

Palavras-chave: culturismo, antropometria, somatótipo, composição corporal, treinamento com pesos.

\begin{abstract}
The aim of this study was to analyze the morphological profile of elite Brazilian bodybuilders who participated in the XXVII Brazilian Championship of Body Building. A total of 28 male athletes (27.0 \pm 7.4 years; 78.2 $\pm 8.7 \mathrm{~kg} ; 170.1 \pm 6.9 \mathrm{~cm}$ ), were voluntarily assessed. Anthropometric measures (body mass, stature, skinfolds thickness, perimeters and diameters) were collected from all subjects in the morning shift, at the competition day. The estimated values of the body composition of the athletes were the following: relative body fat, $5.8 \pm 0.5 \%$; lean body mass, $73.6 \pm 8.2 \mathrm{~kg}$; muscular mass, $54.3 \pm 6.7 \mathrm{~kg}$ and arm muscular area, 108.51 $\pm 15.19 \mathrm{~cm}^{2}$. Conversely, the mean somatotype found was: endomorphy, $1.11 \pm 0.29$; mesomorphy, $8.26 \pm$ 0.67; ectomorphy, $0.95 \pm 0.53$ (balanced mesomorphy). Besides high levels of muscular development ( 70\%) and low body fat buildup, the bodybuilders also presented uniformity in the accumulation of located fat. Predominance of the mesophormic component confirmed muscular structure remarkably developed found in the investigated athletes. Therefore, the results of the present study demonstrate that elite body building athletes present large amount of muscular volume, as well as excellent muscular definition, proportionality and symmetry at the day of the competition.
\end{abstract}

Keywords: body building, anthropometry, somatotype, body composition, weight training.

\section{INTRODUÇÃO}

O culturismo é um esporte que tem como principal característica a aparência física (forma estética), caracterizada pelo perfeito equilíbrio entre volume, proporcionalidade, simetria e definição muscular (ampla visualização dos diferentes grupamentos musculares) ${ }^{(1)}$. Desse modo, os treinamentos dos atletas de culturismo são dinâmicos, realizados sob alta intensidade e com grande exigência de força muscular ${ }^{(2)}$.

Nesse sentido, a intensidade utilizada por culturistas durante as sessões de treinamento com pesos pode variar entre 50 e $85 \%$ de uma repetição máxima (1-RM), dependendo da fase de preparação em que se encontra o atleta ${ }^{(3)}$. Vale ressaltar que a periodização do treinamento no culturismo envolve duas fases distintas, denominadas de fase hipertrófica (fora de temporada) e de definição muscular (pré-competitiva).
Em geral, os atletas de culturismo dedicam a maior parte do ano à fase hipertrófica, na qual a associação entre o treinamento com pesos e o uso de dietas hipercalóricas tem como objetivo o acréscimo de massa muscular. Em contrapartida, a ênfase na definição muscular é a meta principal nos últimos dois ou três meses que antecedem a competição (fase pré-competitiva). Portanto, nesse período, tanto as programações de treinamento quanto a dieta sofrem profundas modificações.

A dieta na fase pré-competitiva, por via de regra, é bastante restritiva, o que provoca importantes alterações nos substratos musculares, nas enzimas, nas proteínas estruturais, bem como nos minerais ${ }^{(4)}$, resultando em redução da força muscular ${ }^{(1)}$. Apesar disso, a intensidade dos treinamentos com pesos deve se manter elevada, evidentemente dentro de limites toleráveis, na tentativa de preservar ao máximo a massa muscular adquirida durante a fase hipertrófica. 
À medida que as competições se aproximam (fase pré-competitiva), o treinamento aeróbio passa a ser incrementado nas sessões de treinamento pela maioria dos culturistas e, conjuntamente com a restrição nutricional e o treinamento de pesos, proporciona ao atleta grande desenvolvimento muscular, com mínimos depósitos de gordura subcutânea e de fluidos.

A utilização de técnicas antropométricas para o monitoramento das modificações morfológicas acarretadas pelas diversas estratégias de preparação adotadas por atletas de culturismo pode proporcionar valiosas informações para o acompanhamento do processo evolutivo dos culturistas, nas diferentes fases de treinamento. Desse modo, medidas de perímetros e de espessuras de dobras cutâneas parecem ser referenciais interessantes a ser utilizados, uma vez que refletem as alterações na musculatura, além de ser de fácil aplicabilidade, boa fidedignidade e baixo custo operacional.

Por outro lado, a utilização do sistema de classificação por somatotipia, embora não reflita as alterações na musculatura, permite boa identificação da estrutura corporal dos atletas de culturismo(2).

Considerando que as informações disponíveis na literatura sobre culturistas em fase pré-competitiva são ainda relativamente incipientes, o propósito deste trabalho foi verificar o perfil morfológico de atletas brasileiros de culturismo de elite, do sexo masculino, que competiram no XXVII Campeonato Brasileiro de Culturismo-Musculação.

\section{METODOLOGIA}

\section{Sujeitos}

De um universo de 82 culturistas do sexo masculino que competiram no XXVII Campeonato Brasileiro de Culturismo-Musculação, 28 atletas (27,0 \pm 7,4 anos) participaram voluntariamente deste estudo. Todos os sujeitos eram campeões em seus respectivos Estados de origem, em diferentes categorias, discriminadas por faixa etária (júnior, sênior e master) e massa corporal (bantam, leve, meio-médio, médio, meio pesado e pesado). Após esclarecidos sobre a proposta do estudo e os procedimentos aos quais seriam submetidos, os participantes assinaram um termo de consentimento livre e esclarecido. Este estudo foi conduzido de acordo com as normas da Resolução 196/96 do Conselho Nacional de Saúde sobre pesquisa envolvendo seres humanos.

\section{Métodos}

Todos os dados foram coletados antes das provas de julgamento (prévias), durante a pesagem oficial dos atletas, no período da manhã.

A massa corporal foi medida em uma balança eletrônica Filizola, com precisão de 0,1 kg, ao passo que a estatura foi determinada em um antropômetro móvel de aço, com precisão de 0,5cm, de acordo com os procedimentos descritos por Gordon et al.(5). A partir das medidas de massa corporal e estatura, o índice de massa corporal (IMC) foi calculado por meio do quociente massa corporal/estatura ${ }^{2}\left(\mathrm{~kg} / \mathrm{m}^{2}\right)$.

A composição corporal foi determinada pela técnica de espessura do tecido celular subcutâneo. Para tanto, três medidas foram coletadas em cada ponto, em seqüência rotacional, do lado direito do corpo, sendo registrado o valor mediano. As espessuras das dobras cutâneas: abdominal, supra-ilíaca, subescapular, tricipital, bicipital, axilar-média, perna-medial e coxa-medial foram medidas por um único avaliador, com um compasso Lange (Cambridge Scientific Instruments, Cambridge, Maryland, EUA), de acordo com as técnicas descritas por Slaughter et al. ${ }^{(6)}$. O coeficiente teste-reteste excedeu
0,95 para cada um dos pontos anatômicos com erro de medida de no máximo $\pm 1,0 \mathrm{~mm}$.

A gordura corporal relativa foi calculada pela fórmula de Brozek et al.(7), a partir da estimativa da densidade corporal determinada pela equação proposta por Lohman ${ }^{(8)}$.

$$
\mathrm{D}(\mathrm{g} / \mathrm{ml})=1,0982-0,000815(\Sigma 3 \mathrm{EDC})+0,0000084(\Sigma 3 \mathrm{EDC})^{2}
$$

onde $\Sigma 3 \mathrm{EDC}=$ somatório da espessura das dobras cutâneas tricipital, subescapular e abdominal.

Os perímetros de peitoral, abdômen, coxa, panturrilha, braço relaxado, braço contraído e antebraço foram medidos com uma fita metálica Lufkin, com precisão de 0,1cm, de acordo com as técnicas convencionais descritas por Callaway et al. ${ }^{(9)}$, com exceção dos perímetros de coxa, mensurado no ponto mesofemoral entre o trocanter maior e a borda superior da patela, e de braço contraído, medido no ponto de maior volume, no final de uma contração voluntária máxima do bíceps. Todas as medidas foram obtidas em duplicata pelo mesmo avaliador, o valor médio sendo utilizado como referência. O coeficiente teste-reteste excedeu 0,97 para cada um dos pontos anatômicos com o erro de medida de no máximo 5\%.

A área muscular do braço (AMB) foi calculada pela seguinte equação ${ }^{(10)}$ :

$$
\operatorname{AMB}\left(\mathrm{cm}^{2}\right)=\left\{[\mathrm{PB}(\mathrm{cm})-\pi \cdot \operatorname{TR}(\mathrm{cm})]^{2} / 4 \cdot \pi\right\}-10
$$

onde PB é o perímetro de braço relaxado e TR é a espessura da dobra cutânea de tríceps.

A massa muscular (MM), por sua vez, foi estimada pela equação proposta por Martin et al. ${ }^{(11)}$.

$$
M M(g)=E\left(0,0553 \cdot P_{C}^{2}+0,0987 \cdot P_{A B R}^{2}+0,0331 \cdot P_{p}^{2}\right)-2.445
$$

onde $\mathrm{E}$ é a estatura, $\mathrm{P}_{C}$ é o perímetro de coxa corrigido pela espessura da dobra cutânea de coxa, $P_{A B R}$ é o perímetro de antebraço e $P_{p}$ é o perímetro de panturrilha corrigido pela espessura da dobra cutânea de perna medial, sendo todas as medidas em centímetros. Os perímetros de coxa e panturrilha foram corrigidos pela simples subtração dos valores encontrados pelo valor de $\pi$ multiplicado pelas respectivas espessuras de dobras cutâneas.

Para a determinação da somatotipia foi utilizado o sistema de classificação do somatótipo de Heath e Carter ${ }^{(12)}$. Para tanto, foram coletados também os diâmetros bicôndilo umeral e bicôndilo femoral, com um compasso de pontas rombas, com precisão de $0,1 \mathrm{~cm}$, de acordo com os procedimentos descritos por Wilmore et al. ${ }^{(13)}$. Essas medidas foram realizadas por um único avaliador, em duplicata, e o coeficiente testereteste excedeu 0,99 para cada um dos pontos anatômicos com o erro de medida de no máximo $\pm 0,1 \mathrm{~cm}$.

Todas as informações foram tratadas mediante procedimentos descritivos por meio do pacote estatístico Statistica ${ }^{T M}$. Os resultados encontrados são apresentados em valores de média, desvio-padrão (DP), erro padrão da média (EPM), intervalo de confiança de 95\% (IC 95\%) e amplitude (valores mínimos e máximos).

\section{RESULTADOS}

As características antropométricas e o somatótipo dos culturistas investigados são apresentados na tabela 1. A grande variação na massa corporal $(78,2 \pm 8,7 \mathrm{~kg})$ e na estatura $(170,1 \pm 6,9 \mathrm{~cm})$ se deve principalmente às diferentes categorias competitivas desses atletas. 
Tabela 1. Características antropométricas de culturistas brasileiros de elite do sexo masculino $(n=28)$

\begin{tabular}{|c|c|c|c|c|c|}
\hline Variáveis & Média & $\mathrm{DP}$ & EPM & IC 95\% & Amplitude \\
\hline Massa corporal (kg) & 78,1 & 8,7 & 1,6 & $74,8-81,5$ & $63,2-96,0$ \\
\hline Estatura $(\mathrm{cm})$ & 170,5 & 6,6 & 1,3 & $167,9-173,1$ & $156,0-180,0$ \\
\hline $\mathrm{IMC}\left(\mathrm{kg} / \mathrm{m}^{2}\right)$ & 27,0 & 1,9 & 0,4 & $26,3-27,8$ & $23,8-30,8$ \\
\hline$\%$ Gordura & 5,8 & 0,5 & 0,1 & $5,6-6,1$ & $4,8-7,3$ \\
\hline$M G(k g)$ & 4,6 & 0,6 & 0,1 & $4,3-4,9$ & $3,6-5,5$ \\
\hline MCM (kg) & 73,6 & 8,2 & 1,5 & $70,1-76,7$ & $59,5-90,5$ \\
\hline MCM/MG & 16,3 & 1,5 & 0,3 & $15,8-16,9$ & $12,6-19,7$ \\
\hline $\mathrm{AMB}\left(\mathrm{cm}^{2}\right)$ & 108,5 & 15,2 & 3,2 & $102,2-115,1$ & $85,9-148,4$ \\
\hline $\mathrm{MM}(\mathrm{kg})$ & 54,3 & 6,7 & 1,4 & $51,3-57,3$ & $42,6-66,8$ \\
\hline$\% \mathrm{MM}$ & 69,7 & 3,3 & 0,7 & $68,3-71,2$ & $58,7-74,6$ \\
\hline \multicolumn{6}{|l|}{$\mathrm{EDC}(\mathrm{mm})$} \\
\hline Abdominal & 4,5 & 1,4 & 0,3 & $4,0-5,1$ & $3,0-10,0$ \\
\hline Supra-ilíaca & 3,1 & 0,7 & 0,1 & $2,8-3,4$ & $2,0-5,5$ \\
\hline Subescapular & 7,4 & 1,2 & 0,2 & $7,0-7,9$ & $4,5-10,0$ \\
\hline Tríceps & 2,9 & 1,1 & 0,2 & $2,5-3,3$ & $1,5-7,0$ \\
\hline Bíceps & 1,5 & 0,6 & 0,1 & $1,3-1,8$ & $0,5-3,5$ \\
\hline Axilar-média & 3,1 & 0,9 & 0,2 & $2,7-3,4$ & $1,5-6,5$ \\
\hline Perna medial & 2,9 & 1,5 & 0,3 & $2,3-3,5$ & $1,5-7,0$ \\
\hline Coxa & 4,5 & 1,7 & 0,3 & $3,9-5,2$ & $2,5-10,0$ \\
\hline Somatório de 8EDC & 30,0 & 6,7 & 1,3 & $27,3-32,6$ & $20,5-52,5$ \\
\hline Média de 8EDC & 3,7 & 0,8 & 0,2 & $3,4-4,1$ & $2,6-6,5$ \\
\hline \multicolumn{6}{|l|}{ Perímetros $(\mathrm{cm})$} \\
\hline Peitoral & 103,6 & 5,9 & 1,2 & $101,1-106,2$ & $89,5-114,2$ \\
\hline Abdominal & 77,3 & 4,6 & 1,0 & $75,3-79,3$ & $68,5-86,5$ \\
\hline Coxa & 59,7 & 3,3 & 0,7 & $58,3-61,1$ & $54,0-64,8$ \\
\hline Panturrilha & 38,6 & 1,8 & 0,4 & $37,8-39,4$ & $35,5-41,4$ \\
\hline Braço relaxado & 39,5 & 2,4 & 0,5 & $38,4-40,5$ & $35,5-45,5$ \\
\hline Braço contraído & 41,1 & 2,3 & 0,5 & $40,0-42,1$ & $37,0-47,5$ \\
\hline Antebraço & 31,0 & 1,3 & 0,3 & $30,4-31,6$ & $28,5-35,0$ \\
\hline \multicolumn{6}{|c|}{ Diâmetros ósseos (cm) } \\
\hline Bicôndilo do úmero & 7,5 & 0,5 & 0,1 & $7,3-7,7$ & $7,0-8,0$ \\
\hline Bicôndilo do fêmur & 9,7 & 0,6 & 0,1 & $9,5-9,9$ & $9,0-11,0$ \\
\hline \multicolumn{6}{|l|}{ Somatótipo } \\
\hline Endomorfia & 1,11 & 0,29 & 0,06 & $0,99-1,23$ & $0,57-1,74$ \\
\hline Mesomorfia & 8,26 & 0,67 & 0,24 & $7,78-8,74$ & $7,21-9,73$ \\
\hline Ectomorfia & 0,95 & 0,53 & 0,05 & $0,85-1,05$ & $0,01-2,20$ \\
\hline
\end{tabular}

Nota. DP = desvio-padrão; EPM = erro padrão da média; IC 95\% = intervalo de confiança de 95\%; $M G$ = massa de gordura; $M C M=$ massa corporal magra; $A M B=$ área muscular do braço; $\mathrm{MM}=$ massa muscular.
Os resultados indicaram baixos níveis de gordura corporal ( 6\%), com variação de 2,5\% entre os valores extremos; o acúmulo médio de gordura subcutânea observado nos diferentes pontos anatômicos foi de $\sim 4 \mathrm{~mm}$. Nesse sentido, os pontos de maior e menor acúmulo foram as regiões subescapular e bicipital, respectivamente $(5,9 \mathrm{~mm}$ de variação média entre elas). Por outro lado, os valores elevados de mesomorfia confirmaram o elevado nível de massa muscular ( 70\%) encontrado nos culturistas do presente estudo.

\section{DISCUSSÃO}

A maior dificuldade para analisar os resultados encontrados em estudos com culturistas é que parte das investigações não relata exatamente a fase de treinamento na qual se encontravam os atletas no momento da coleta de dados. Além disso, o uso de diferentes técnicas de medidas para as variáveis antropométricas não favorece análise mais precisa ou, ainda, comparação mais criteriosa dos resultados apresentados pelos estudos disponíveis na literatura que se propuseram a investigar atletas dessa modalidade.

Apesar dessas limitações, informações disponíveis na literatura sobre a quantidade de gordura corporal relativa de atletas de culturismo do sexo masculino são apresentadas na tabela 2. Embora muitos dos estudos revisados não precisem com exatidão o momento em que foram coletados os dados, acredita-se que valores entre 4 e $10 \%$ sejam representativos do período pré-competitivo.

Com base nos estudos que adotaram o método de espessura de dobras cutâneas para a avaliação da composição corporal de culturistas, observa-se falta de uniformidade com relação ao tipo de compasso utilizado, bem como na escolha dos modelos matemáticos para a estimativa da densidade corporal e da gordura corporal relativa, o que pode acarretar importantes diferenças na estimativa dos componentes da composição corporal(14).

No presente estudo, bem como em grande parte das investigações realizadas com atletas de culturismo, foi empregado o método de espessura de dobras cutâneas para a estimativa da gordura corporal relativa. A grande vantagem da utilização desse método em atletas dessa modalidade é que o conhecimento dos depósitos de gordura subcutânea proporciona importantes informações relativas ao grau de definição muscular em que se encontram esses sujeitos, principalmente no período pré-competitivo. Baseados nessas informações, os culturistas podem estar corrigindo, periodicamente, as possíveis deficiências nas estratégias adotadas nos diferentes períodos de preparação, tanto com relação ao treinamento quanto aos aspectos dietéticos.

Com relação à equação escolhida para a estimativa da densidade corporal dos atletas nesta investigação, optou-se por aquela que, aparentemente, é a mais adequada para o uso em culturistas competitivos, uma vez que não existem equações antropométricas desenvolvidas especificamente para essa população. A equação utilizada neste estudo foi validada anteriormente, por meio do método de pesagem hidrostática, em uma amostra de 12 culturistas norte-americanos; foi a que melhor atendeu aos critérios de validação quando comparada com outras 19 equações analisadas( ${ }^{(15)}$.

Os valores individuais de espessura das oito dobras cutâneas mensuradas foram inferiores aos encontrados em outras investigações semelhantes ${ }^{(1,16-18)}$; contudo, o comportamento dos acúmulos regionais de gordura foi bastante similar, sendo predominante nas regiões subescapular, coxa, abdominal e supra-ilíaca. Nesse sentido, Fry et al.(16) afirmam que, em culturistas, os depósitos adiposos localizados na re- 
Tabela 2. Valores estimados de gordura corporal relativa de culturistas do sexo masculino encontrados em diferentes estudos

\begin{tabular}{|c|c|c|c|c|c|c|c|c|}
\hline Investigadores & $\mathrm{n}$ & $\begin{array}{l}\text { Idade } \\
\text { (anos) }\end{array}$ & Fase de treinamento & $\begin{array}{c}\text { \% Gordura } \\
\text { corporal }\end{array}$ & Método & $\begin{array}{c}\% \text { Gordura corporal } \\
\text { (equação) }\end{array}$ & $\begin{array}{c}\text { Densidade corporal } \\
\text { (equação) }\end{array}$ & $\begin{array}{l}\text { Tipo de } \\
\text { compasso }\end{array}$ \\
\hline Alway et al. ${ }^{(37)}$ & 5 & $32,8 \pm 2,0$ & NR & $13,0 \pm 5,1$ & $\mathrm{PH}$ & $\operatorname{Siri}^{(40)}$ & - & - \\
\hline \multirow[t]{2}{*}{ Bamman et al. ${ }^{(1)}$} & 6 & 26,5 & Pré-competitiva & $4,1 \pm 1,3$ & $\mathrm{PH}$ & Brozek et al..$^{(7)}$ & - & - \\
\hline & & & & 5,1 & EDC & $\operatorname{Sir} i^{(40)}$ & Jackson \& Pollock ${ }^{(41)}$ & Harpenden \\
\hline Bazzarre et al. ${ }^{(38)}$ & 19 & $28 \pm 4$ & Pré-competitiva & $6,0 \pm 1,8$ & EDC & $\operatorname{Siri}(40)$ & Jackson \& Pollock ${ }^{(41)}$ & NR \\
\hline Bazzarre et al. ${ }^{(39)}$ & 13 & $30 \pm 4$ & Pré-competitiva & $4,9 \pm 1,6$ & EDC & $\operatorname{Sir} i^{(40)}$ & Jackson \& Pollock ${ }^{(41)}$ & Lange \\
\hline \multirow[t]{2}{*}{ Elliot et al. ${ }^{(19)}$} & 16 & $25 \pm 5$ & Pré-competitiva & $7,2 \pm 1,9$ & $\mathrm{PH}$ & Brozek et al..$^{(7)}$ & - & - \\
\hline & & & & $6,1 \pm 2,0$ & EDC & $\operatorname{Sir} i^{(40)}$ & Jackson \& Pollock ${ }^{(41)}$ & Harpenden \\
\hline Faber et al. (22) & 76 & $27,4 \pm 6,5$ & Hipertrofia & $15,4 \pm 2,9$ & EDC & $\operatorname{Sir} i^{(40)}$ & Durnin \& Rahaman ${ }^{(42)}$ & Harpenden \\
\hline Fahey et al. ${ }^{(20)}$ & 2 & $29,0 \pm 7,1$ & NR & $8,4 \pm 3,9$ & $\mathrm{PH}$ & Brozek et al..$^{(7)}$ & - & - \\
\hline Fry et al. ${ }^{(16)}$ & 36 & $24,6 \pm 4,8$ & Pré-competitiva & $9,3 \pm 1,6$ & EDC & Brozek et al..$^{(7)}$ & Lohman ${ }^{(8)}$ & Lange \\
\hline Hurley et al.(33) & 8 & $29 \pm 1$ & NR & $12 \pm 1$ & $\mathrm{PH}$ & Brozek et al..$^{(7)}$ & - & - \\
\hline Katch et al. ${ }^{(25)}$ & 18 & $27,8 \pm 1,8$ & NR & $9,3 \pm 0,8$ & $\mathrm{PH}$ & $\operatorname{Sir} r^{(40)}$ & - & - \\
\hline Sandoval et al. ${ }^{(27)}$ & 5 & $25,0 \pm 3,3$ & Pré-competitiva & $7,2 \pm 1,6$ & $\mathrm{PH}$ & Brozek et al..$^{(7)}$ & - & - \\
\hline Silva et al. ${ }^{(17)}$ & 23 & $33,4 \pm 11,4$ & Pré-competitiva & $9,7 \pm 0,5$ & EDC & - & Faulkner* & Lange \\
\hline Spitler et al. ${ }^{(28)}$ & 10 & $30,1 \pm 8,1$ & NR & $9,9 \pm 1,9$ & $\mathrm{PH}$ & Brozek et al..$^{(7)}$ & - & - \\
\hline \multirow[t]{3}{*}{ Withers et al. ${ }^{(18)}$} & 3 & $26,3 \pm 4,7$ & Pré-competitiva & $7,1 \pm 2,0$ & $\mathrm{PH}$ & Brozek et al..$^{(7)}$ & - & - \\
\hline & & & & $5,5 \pm 0,3$ & M3C & - & - & - \\
\hline & & & & $5,0 \pm 0,2$ & $\mathrm{M} 4 \mathrm{C}$ & - & - & \\
\hline Presente estudo & 28 & $27,0 \pm 7,4$ & Pré-competitiva & $5,8 \pm 0,5$ & EDC & Brozek et al..$^{(7)}$ & Lohman ${ }^{(8)}$ & Lange \\
\hline
\end{tabular}

Nota. $\mathrm{NR}=$ não relatado; $\mathrm{PH}$ = pesagem hidrostática; $\mathrm{EDC}=$ espessura de dobras cutâneas; $\mathrm{M} 3 \mathrm{C}=$ modelo de três compartimentos; $\mathrm{M} 4 \mathrm{C}=$ modelo de quatro compartimentos; *referência não localizada na literatura.

gião posterior do tronco parecem ser os últimos a se reduzir durante o processo de definição muscular.

Analisando-se os dados do presente estudo (tabela 1), verifica-se que os culturistas, no dia da competição, apresentaram acentuado desenvolvimento muscular, com reduzidos depósitos de gordura corporal, perfil diferenciado do encontrado na grande maioria dos atletas e/ou praticantes de outras modalidades esportivas ou, ainda, de não-atletas ${ }^{(19,20)}$.

Apesar da baixa quantidade de gordura relativa, a grande massa muscular absoluta dos atletas investigados resultou em IMC = 27kg/ $\mathrm{m}^{2}$. Esse valor, quando analisado de forma isolada, pode resultar em interpretações equivocadas sobre a real condição atlética desses indivíduos. Isso vem ao encontro dos achados de outros estudos que têm demonstrado que o IMC é um índice bastante limitado para estimar a composição corporal, principalmente em atletas envolvidos em treinamentos de força, uma vez que esse índice não discrimina se o sobrepeso determinado ocorre em razão da grande massa muscular ou do excesso de gordura corporal|(18,21-23).

Portanto, embora o IMC seja um índice freqüentemente utilizado para analisar a prevalência de sobrepeso ou obesidade, em diferentes populações, sua validade deve ser questionada, sobretudo, em estudos onde a população analisada inclui indivíduos com elevados níveis de massa muscular.

A massa muscular dos atletas estudados representou cerca de $69,7 \%$ da massa corporal total ou ainda $74,1 \%$ da massa corporal magra. Esses valores são superiores (cerca de 6,6 a 7,2\%, respectivamente) aos encontrados por Spenst et al.(2), em 10 culturistas canadenses, e por Cyrino et al. ${ }^{(24)}$, em seis culturistas brasileiros. Provavelmente, os menores valores de massa muscular encontrados por esses dois estudos sejam produto de maior quantidade de gordura corporal total, associada a maior retenção hídrica subcutânea, bastante comum em culturistas durante a fase de treinamento para hipertrofia muscular, visto que nessa fase são utilizadas, freqüentemente, dietas hipercalóricas na tentativa de promover o aumento do volume muscular.

Vale ressaltar que. para o cálculo da massa muscular dos atletas dos estudos apresentados anteriormente, bem como dos culturistas do presente estudo, foi utilizada uma equação preditiva desenvolvida a partir da dissecação de 12 cadáveres do sexo masculino, de 50 a 94 anos. Embora essa equação tenha fornecido estimativas bastante consistentes durante sua validação(11) $\left(E P E=1,58 \mathrm{~kg} ; r^{2}=0,93\right)$, 
não se podem descartar as limitações de sua aplicação em outras amostras. Entretanto, considerando que as comparações entre os estudos com atletas de culturismo foram realizadas com base em informações obtidas de forma semelhante, o erro produzido passa a ser sistemático.

A relação entre a massa corporal magra/massa de gordura verificada neste estudo foi muito inferior (31\%) à encontrada por Bamman et al. (1), no final de um estudo que acompanhou seis culturistas de elite durante 12 semanas anteriores a uma competição; a quantidade de gordura corporal relativa dos atletas investigados por esses pesquisadores (4,1\%), estimada por pesagem hidrostática, também foi inferior à encontrada nos atletas do presente estudo. Todavia, nesse mesmo estudo, quando os autores determinaram a estimativa da gordura corporal relativa pelo método de espessura de dobras cutâneas, o valor médio encontrado $(5,1 \%)$ foi mais próximo daquele observado nesta investigação $(5,8 \%)$, reduzindo as diferenças na razão entre a massa corporal magra/massa de gordura para aproximadamente 14\%.

Tanto o valor médio encontrado no presente estudo quanto o relatado por Bamman et al.(1) para a razão entre a massa corporal magra/massa de gordura (16,29 e 23,62, respectivamente) são superiores aos revelados por Katch et al. ${ }^{(25)}(9,7)$, Pipes $^{(26)}(11,1)$ e Sandoval et al. ${ }^{(27)}$ $(12,9)$, em outros estudos realizados com culturistas. Provavelmente, essas diferenças podem ter sido acarretadas pelas mudanças estabelecidas nos critérios de julgamento das competições de culturismo ao longo dos tempos.

Nesse sentido, o principal ponto de análise nas competições de culturismo da década de 1960 até o início da década de 1980 residia primordialmente sobre o tamanho muscular, os atletas competindo com grandes volumes musculares, entretanto, com pouca definição muscular. A maioria dos atletas acreditava que o sucesso nessa modalidade estava atrelado ao excesso de treinamento(25) e ao uso de dietas hipercalóricas, principalmente à base de alimentos de alto teor protéico ${ }^{(28)}$. Todavia, pouco se conhecia sobre os reais efeitos da adoção dessas estratégias e os culturistas baseavam sua preparação para as competições nos relatos de outros atletas mais experientes e bemsucedidos nessa modalidade.

A partir daí, os critérios de julgamento adotados até então para as competições de culturismo começaram a ser questionados, o que resultou em mudança do paradigma vigente, a avaliação dos níveis de definição muscular passando a ocupar lugar de destaque no julgamento dos atletas durante as competições. Assim, os atletas passaram a competir mais bem definidos, contudo, com redução acentuada do volume muscular, uma vez que muitos deles passaram a adotar restrições nutricionais extremamente severas, sobretudo, durante as últimas duas ou três semanas que antecediam as competições.

Somente no final da década de 1980 e no início da década de 1990 é que se chegou a um consenso de que o sucesso dos atletas de culturismo durante as competições deveria estar associado a perfeita combinação entre o máximo volume muscular, ótima definição, proporcionalidade e simetria. Assim, o acompanhamento de diferentes aspectos da preparação do atleta passou a ser o grande diferencial entre o sucesso e o fracasso nessa modalidade.

Com base nessas informações torna-se mais fácil, pelo menos aparentemente, compreender as possíveis causas das diferenças morfológicas verificadas entre os grupos de culturistas investigados em estudos realizados nas diferentes décadas.
Estudos disponíveis na literatura sobre culturistas têm demonstrado que durante o período pré-competitivo existe diminuição nos perímetros da maioria dos grupamentos musculares, provocada principalmente pela redução nos depósitos de gordura subcutânea ${ }^{(1,18)}$. Infelizmente, no presente estudo, as condições prévias dos competidores não foram acompanhadas, o que desfavorece uma análise mais criteriosa sobre os resultados apresentados pelos atletas investigados no dia da competição.

Os valores dos diâmetros bicôndilo umeral e bicôndilo femoral apresentados pelos culturistas neste estudo foram ligeiramente superiores aos relatados por Silva et al. ${ }^{(17)}$, em amostra composta por 23 culturistas brasileiros de elite, com características semelhantes às do presente estudo. Entretanto, os valores encontrados em ambos os estudos foram inferiores aos reportados por Spitler et al.(28), o que é plenamente justificável pelas diferenças nas dimensões corporais dos grupos de culturistas investigados.

Por outro lado, o somatótipo médio estabelecido para os culturistas na presente investigação (mesomorfos balanceados) corrobora os achados de Silva ${ }^{(17)}$, em culturistas brasileiros, bem como os de Borms et al.(29), em competidores do Campeonato Mundial Amador de Culturismo-Musculação (Mister Universo) realizado na cidade do Cairo, no Egito, em 1981. Os valores encontrados (1,11 - 8,26 - 0,95) evidenciaram o grande desenvolvimento muscular e os baixos níveis de gordura dos atletas deste estudo. Anteriormente, Spitler et al.(28) já haviam observado um perfil semelhante em 19 culturistas competitivos, o que sugere que esse parece ser o padrão mais adequado para o sucesso nessa modalidade.

Os baixos valores do componente endomorfo, bem como os depósitos de gordura corporal extremamente baixos, observados em culturistas têm sido verificados predominantemente em situações que envolvem restrição calórica associada a treinamentos com pesos e, na maioria das vezes, exercícios aeróbios ${ }^{(1,27,30)}$. Logo, essa condição é estabelecida somente durante algumas semanas ou poucos meses ao longo de uma temporada competitiva (fase pré-competitiva e competitiva propriamente dita). A adoção dessa estratégia parece ser eficiente, sobretudo, quando empregada na fase de definição muscular, uma vez que a combinação entre treinamentos concorrentes (força muscular e resistência aeróbia), pelo menos aparentemente, não favorece o desenvolvimento muscular acentuado buscado pelos culturistas durante a fase hipertrófica ${ }^{(30-32)}$.

Adicionalmente, outros recursos também têm sido amplamente utilizados por culturistas na tentativa de otimizar ainda mais os processos de hipertrofia e definição muscular. Nesse sentido, a administração de agentes farmacológicos entre esses atletas, particularmente esteróides anabólicos e diuréticos, tem-se popularizado ao longo dos $\operatorname{anos}^{(1,19,33-36)}$.

Vale ressaltar, ainda, que os baixos níveis de gordura subcutânea favorecem o julgamento durante uma competição de culturismo, visto que proporcionam observação mais detalhada do tamanho muscular, da vascularização, da separação entre os diferentes músculos, das estriações específicas de cada grupo muscular, da proporcionalidade e da simetria.

\section{CONCLUSÕES}

Os atletas de culturismo do presente estudo apresentaram composição corporal pouco usual para a maioria dos atletas de diferentes modalidades esportivas, sendo caracterizada por elevados níveis de massa muscular ( 70\%) e baixos depósitos de gordura corporal ( 6\%). 
A predominância do componente mesomorfo confirmou a estrutura muscular acentuadamente desenvolvida encontrada nos atletas investigados.

Além disso, o estudo mostrou que o IMC é um índice bastante limitado para análise das características morfológicas da maioria dos atletas de culturismo. Nesse sentido, com base somente nos valores produzidos pelo IMC, muitos dos atletas investigados seriam classificados como portadores de sobrepeso ou, até mesmo, de obesidade, quando na verdade possuem quantidades de gordura corporal extremamente baixas. Portanto, no caso dos atletas dessa modalidade, o IMC não consegue discriminar se os elevados valores produzidos são decorrentes do acúmulo de gordura corporal ou do excesso de massa muscular.

\section{REFERÊNCIAS BIBLIOGRÁFICAS}

1. Bamman MM, Hunter GR, Newton LE, Roney RK, Khaled MA. Changes in body composition, diet, and strength of bodybuilders during the 12 weeks prior to competition. J Sports Med Phys Fitness 1993;33:383-91.

2. Spenst LF, Martin AD, Drinkwater DT. Muscle mass of competitive male athletes. J Sports Sci 1993;11:3-8.

3. Cyrino ES. Efeito da suplementação protéica sobre indicadores metabólicos de fadiga em atletas de culturismo. Rio Claro, 1997. Dissertação (Mestrado) - Unesp, Instituto de Biociências.

4. Russell DM, Walker PM, Leiter LA, Sima AA, Tanner WK, Mickle DA, et al. Metabolic and structura changes in skeletal muscle during hypocaloric dieting. Am J Clin Nutr 1984;39:503-13.

5. Gordon CC, Chumlea WC, Roche AF. Stature, recumbent length, and weight. In: Lohman TG, Roche AF, Martorell R, editors. Anthropometric standardizing reference manual. Champaign: Human Kinetics, 1988;3-8.

6. Slaughter MH, Lohman TG, Boileau RA, Stillman RJ, Van Loan M, et al. Influence of maturation on relationship of skinfolds to body density: a cross-sectional study. Hum Biol 1984;56:681-9.

7. Brozek J, Grande F, Anderson JT, Keys A. Densitometric analysis of body composition: revision of some quantitative assumptions. Ann N Y Acad Sci 1963;110:113-40

8. Lohman TG. Skinfolds and body density and their relation to body fatness: a review. Hum Biol 1981;53:181-225.

9. Callaway CW, Chumlea WC, Bouchard C, Himes JH, Lohman TG, et al. Circumferences. In: Lohman TG, Roche AF, Martorell R., editors. Anthropometric standardizing reference manual. Champaign: Human Kinetics, 1988;39-54

10. Frisancho AR. New standards of weight and body composition by frame size and height for assessment of nutritional status of adults and the elderly. Am J Clin Nutr 1984;40:808-19

11. Martin AD, Spenst LF, Drinkwater DT, Clarys JP. Anthropometric estimation of muscle mass in men. Med Sci Sports Exerc 1990;22:729-33.

12. Heath BH, Carter JEL. A modified somatotype method. Am J Phys Anthropol 1967;27:57-74.

13. Wilmore JH, Frisancho RA, Gordon CC, Himes JH, Martin AD, et al. Body breadth equipment and measurement techniques. In: Lohman TG, Roche AF, Martorell R, editors. Anthropometric standardizing reference manual. Champaign: Human Kinetics, 1988;27-38.

14. Cyrino ES, Okano AH, Glaner MF, Romanzini M, Gobbo LA, et al. Impacto da utilização de diferentes compassos de dobras cutâneas para a análise da composição corporal. Rev Bras Med Esporte 2003:9:145-9.

15. Fry AC, Cisar CJ, Housh TJ. A comparison of anthropometric equations for estimating body density in male competitive body builders. J Appl Sport Sci Res 1987;1:61-5.

16. Fry AC, Ryan AJ, Schwab RJ, Powell DR, Kraemer WJ. Anthropometric characteristics as discriminators of body-building success. J Sports Sci 1991;9:23-32

17. Silva PRP, Trindade RS, De Rose EH. Composição corporal, somatotipo e proporcionalidade de culturistas de elite do Brasil. Rev Bras Med Esporte 2003;9:403-7.

18. Withers RT, Noell CJ, Whittinghan NO, Chatterton BE, Schultz CG, Keeves JP. Body composition changes in elite male bodybuilders during preparation for competition. Aust J Sci Med Sport 1997;29:11-6.

19. Elliot DL, Goldberg L, Kuehl KS, Catlin DH. Characteristics of anabolic-androgenic steroid-free male and female bodybuilders. Phys Sportsmed 1987;15:169-79.

20. Fahey TD, Akka L, Rolph R. Body composition and $\mathrm{VO}_{2}$ max of exceptional weight-trained athletes. J Appl Physiol 1975:39:559-61.
Portanto, os resultados do presente estudo demonstraram que atletas de culturismo de elite apresentam no dia da competição grande volume muscular acompanhado por excelente definição muscular, proporcionalidade e simetria.

\section{AGRADECIMENTOS}

Os autores agradecem ao CNPq, a Capes e a FAPESP pelas bolsas concedidas.

Todos os autores declararam não haver qualquer potencial conflito de interesses referente a este artigo.
21. Maestá N, Cyrino ES, Nardo Júnior N, Morelli MYG, Santarém JM, Burini RC. Antropometria de atletas culturistas em relação à referência populacional. Rev Nutr 2000;13:135-41.

22. Faber M, Benadé AJS, Van Eck M. Dietary intake, anthropometric measurements, and blood lipid values in weight training athletes (body builders). Int J Sports Med 1986;7:342-6.

23. Kleiner SM, Bazzarre TL, Ainsworth BE. Nutritional status of nationally ranked elite bodybuilders. Int J Sport Nutr 1994;4:54-69.

24. Cyrino ES, Maestá N, Burini RC. Aumento de força e massa muscular em atletas de culturismo suplementados com proteína. Trein Desp 2000;5:9-18.

25. Katch VL, Katch Fl, Moffatt R, Gittleson M. Muscular development and lean body weight in body builders and weight lifters. Med Sci Sports Exerc 1980;12:340-4.

26. Pipes TV. Physiologic characteristics of elite body builders. Phys Sportsmed 1979;7:116-20

27. Sandoval WM, Heyward VH, Lyons TM. Comparison of body composition, exercise and nutritional profiles of female and male body builders at competition. J Sports Med Phys Fitness 1989;29:63-70.

28. Spitler DL, Dias FJ, Horvath SM, Wright JE. Body composition and maximal aerobic capacity of bodybuilders. J Sports Med Phys Fitness 1980;20:181-8.

29. Borms J, Ross WD, Duquet W, Carter JEL. Somatotypes of world class bodybuilders. In: Day JAP, editors. Perspectives in kinanthropometry. Champaign: Human Kinetics, 1986;81-90.

30. Dudley GA, Fleck SJ. Strength and endurance training. Are they mutually exclusive? Sports Med 1987;4:79-85.

31. Dudley GA, Djamil R. Incompatibility of endurance and strength training modes of exercise. J Appl Physiol 1985:59:1446-51.

32. Hickson RC. Interference of strength development by simultaneously training for strength and endurance. Eur J Appl Physiol 1980;45:255-63.

33. Hurley BF, Seals DR, Hagberg J, Goldberg AC, Ostrove SM, Holloszy JO, et al. High-density-lipoprotein cholesterol in bodybuilders vs. powerlifters. Negative effects of androgen use. JAMA 1984;252:507-13

34. Keith RE, Stone MH, Carson RE, Lefavi RG, Fleck SJ. Nutritional status and profiles of trained steroidusing bodybuilders. Int J Sport Nutr 1996;6:247-54.

35. Kleiner SM, Bazzarre TL, Lichford MD. Metabolic profiles, diet, and health practices of championship male and female bodybuilders. J Am Diet Assoc 1990;90:962-7.

36. Sturmi JE, Rutecki GW. When competitive bodybuilders collapse. A result of hyperkalemia? Phys Sportsmed 1995;23:49-53.

37. Always SE, Grumbt WH, Stray-Gundersen J, Gonyea WJ. Effects of resistance training on elbow flexors of highly competitive bodybuilders. J Appl Physiol 1992;72:1512-21

38. Bazzarre TL, Kleiner SM, Litchford MD. Nutrient intake, body fat, and lipid profiles of competitive male and female bodybuilders. J Am Coll Nutr 1990;9:136-42.

39. Bazzarre $T L$, Kleiner SM, Ainsworth BE. Vitamin C intake and lipid profiles of competitive male and female bodybuilders. Int J Sport Nutr 1992;2:260-71

40. Siri WE. Body composition from fluid space and density. In: Brozek J, Hanschel A., editors. Techniques for measuring body composition. Washington: National Academy of Science, 1961;223-44.

41. Jackson AS, Pollock ML. Generalized equations for predicting body density of men. Brit J Nutr 1978:40:497-504

42. Durnin JV, Rahaman MM. The assessment of the amount of fat in the human body from measurements of skinfold thickness. Brit J Nutr 1967:21:681-9. 